\title{
Signal Restoration from Data Aliased in Time
}

\author{
KUMAR SWAMINATHAN, STUDENT MEMBER, IEEE
}

\begin{abstract}
This paper deals with the problem of signal restoration from data aliased in time. The signal, which is in general noncausal, is split into a causal and an anticausal part. The causal part and the timereversed anticausal part are then modeled as impulse responses of rational pole-zero models. The parameters of these models are then estimated by solving sets of overdetermined equations. The choice of model orders, i.e., number of poles and zeros of rational models, is also discussed. It is shown that if the aliasing period is large enough, there is sufficient information for all the parameters to be estimated. The special cases of a purely causal signal or a purely anticausal signal are discussed. Simulation results show that the signal can be recovered with excellent accuracy.
\end{abstract}

\section{INTRODUCTION}

$\mathbf{I}_{\mathrm{ver}}^{\mathrm{N}}$ $\mathrm{N}$ signal processing, one encounters the problem of aliasing very frequently. Aliasing is a phenomenon usually associated with the frequency domain. If a continuous signal $s(t)$ has a Fourier transform $S(f)$, then the phenomenon in which frequency-shifted versions of $S(f)$ overlap is called aliasing. One can easily extend this definition to define aliasing in the time domain. Thus, aliasing in the continuous time domain is the phenomenon in which time-shifted versions of $s(t)$ overlap. Similarly, aliasing in the discrete time domain is the phenomenon in which time-shifted versions of the sequence $s(n)$ overlap.

Signal restoration from data aliased in the frequency domain is discussed in [1] for continuously sampled band-limited signals. Several other references relating to the signal restoration for the frequency domain aliased data have also been cited in [1]. In this paper, we concentrate on the problem of signal restoration from data aliased in time. We will focus our attention only on the discrete case.

Aliasing in the time domain arises in several situations. For example, it could arise due to inadequate sampling in the frequency domain followed by the inverse Fourier transform. Thus, if the signal $s(t)$ were sampled in the frequency domain every $F \mathrm{~Hz}$, then the inverse Fourier transform of the sampled signal would be given by

$$
h(t)=\sum_{n=-\infty}^{\infty} s(t+n / F)
$$

Manuscript received March 28, 1983; revised November 14, 1983, March 5,1984, and June ?, 1984. This work was performed while the author was supported as a graduate student at the California Institute of Technology, Pasadena. The facility used was the Caltech Psychoacoustic Signal Processing Facility, which is funded in part by the Jet Propulsion Laboratory, and operated by Caltech for the National Aeronau tics and Space Administration under Contract NAS7-918.

The author is with the Department of Electrical Engineering, California Institute of Technology, Pasadena, CA 91125. which is an aliased version of $s(t)$. Aliasing in the time domain also arises when we wish to estimate the impulse response of a linear system from the system response to a periodic train of impulses. Here the signal restoration problem takes the form of a linear system identification problem. An example of this would be the deconvolution of voiced speech, which can be represented as the output of a frequency-shaping filter when excited by a train of impulses [21], [22]. The linear system that we wish to identify in this example is the frequencyshaping filter. In all of these cases, one can apply the signal restoration algorithm.

In the discrete time domain, we can express the aliased data $h(n)$ as

$$
h(n)=\sum_{r=-\infty}^{\infty} s\left(n+r n_{0}\right)
$$

where $s(n)$ is the signal that needs to be recovered and $n_{0}$ is the aliasing period. In this paper, we will assume that there is no noise and that the value of $n_{0}$ is known exactly. Without any information at all about the signal $s(n)$, it cannot be recovered uniquely from the aliased data. But in most situations, we have some a priori information about $s(n)$. This information could be in the form of a model for $s(n)$. If $n_{0}$ is large enough, then the aliased data may have enough information so that all the parameters of the model can be estimated.

In this paper, it is assumed that the signal $s(n)$ does obey a given model. The signal is, in general, assumed to be noncausal, i.e., $s(n)$ takes on nonzero values from $n=-\infty$ to $n=$ $+\infty$. The noncausal signal is then split into a causal and an anticausal part. Both the causal part and the time-reversed anticausal part are then modeled as the impulse response of rational pole-zero models. The signal restoration problem now reduces to the problem of estimating the parameters of these models for which algorithms are described.

The algorithms presented in this paper are related to the work done in the area of digital filter design from time domain specifications. The most relevant work is the paper due to Burrus and Parks [2]. Our work is largely an extension of some of the optimal approximate methods for digital filter synthesis described in [2]. Another very relevant paper is by Shanks [8]. Several other contributions in this area are listed in [9]-[13]. Some of the techniques used in this paper are also similar to the well-known LPC techniques which have been very successfully applied in several areas such as speech coding, seismic signal processing, and spectral estimation. A tutorial review of linear prediction is given by Makhoul [14]. Several other references which concentrate on applications are listed in [15]-[17]. 
The organization of the paper is as follows. In Section I, we review the Burrus-Parks algorithm. In Section II, we describe the signal model and show how the aliased data can be related to this model. In Section III, we describe algorithms for determining the predictor polynomials that characterize the signal, In Section IV, technqiues for estimating the numerator polynomials of the signal model are described. Simulation results are presented in Section V, and a brief summary and conclusions are presented in Section VI.

\section{Review of Burrus-Parks Algorithm}

In this section, we briefly review the work due to Burrus and Parks. In their paper, Burrus and Parks concentrate on the problem of designing a filter $G(z)$ where

$$
G(z)=\frac{C(z)}{B(z)}=\frac{\sum_{i=0}^{N} c_{i} z^{-i}}{1+\sum_{i=1}^{M} b_{i} z^{-i}}
$$

so that its impulse response $g(n)$ matches a given sequence $h(n)$ over the range of $n=0,1, \cdots, n_{0}-1$. Several approximate methods have been proposed to solve this problem. We will focus our attention only on the modified or indirect approximate methods.

Equation (1) can be expressed in terms of convolution as

$$
\begin{aligned}
g_{n}+\sum_{i=1}^{M} b_{i} g_{n-i} & =c_{n} & & n=0,1, \cdots, N \\
& =0 & & n>N
\end{aligned}
$$

or in matrix form

$$
\left[\begin{array}{c}
c_{0} \\
c_{1} \\
\vdots \\
c_{N} \\
0 \\
\vdots \\
0
\end{array}\right]=\left[\begin{array}{cccc}
g_{0} & 0 & \cdots & 0 \\
g_{1} & g_{0} & \cdots & \\
\vdots & \vdots & & \\
g_{N} & g_{N-1} & \cdots & \\
g_{N+1} & g_{N} & \cdots & \\
\vdots & \vdots & & \\
g_{n_{0}-1} & g_{n_{0}-2} & \cdots & g_{n_{0}-M-1}
\end{array}\right]\left[\begin{array}{c}
1 \\
b_{1} \\
\\
b_{M}
\end{array}\right] .
$$

One now replaces the unknown sequence $g(n)$ by the given sequence $h(n)$ in (2). The exact equation (2) is thus modified to define the error terms $\epsilon_{0}, \epsilon_{1}, \cdots, \epsilon_{n_{0}-1}$.

$$
\left[\begin{array}{c}
c_{0}+\epsilon_{0} \\
c_{1}+\epsilon_{1} \\
\vdots \\
c_{N}+\epsilon_{N} \\
\epsilon_{N+1} \\
\vdots \\
\epsilon_{n_{0}-1}
\end{array}\right]=\left[\begin{array}{cccc}
h_{0} & 0 & \cdots & 0 \\
h_{1} & h_{0} & \cdots & \\
\vdots & \vdots & & \\
h_{N} & h_{N-1} & \cdots & \\
h_{N+1} & h_{N} & \cdots & \\
\vdots & \vdots & & \\
h_{n_{0}-1} & h_{n_{0}-2} & \cdots & h_{n_{0}-M-1}
\end{array}\right]\left[\begin{array}{c}
1 \\
b_{1} \\
\\
b_{M}
\end{array}\right]
$$

From this, one obtains

$$
\left[\begin{array}{c}
\epsilon_{N+1} \\
\epsilon_{N+2} \\
\vdots \\
\epsilon_{n_{0}-1}
\end{array}\right]=\left[\begin{array}{c}
h_{N+1} \\
h_{N+2} \\
\vdots \\
h_{n_{0}-1}
\end{array}\right]+\left[\begin{array}{lll}
h_{N} & \cdots & \\
h_{N+1} & \cdots & \\
h_{n_{0}-2} & \cdots & h_{n_{0}-M-1}
\end{array}\right]\left[\begin{array}{c}
b_{1} \\
b_{2} \\
b_{M}
\end{array}\right]
$$

or in more compact notation

$$
\hat{\epsilon}=\hat{h}+H b .
$$

Assuming that $n_{0} \geqslant M+N+1$, we can find the vector $\boldsymbol{b}$ which minimizes $\hat{\epsilon}^{T} \hat{\epsilon}$. This can either be done by singular value decomposition of $\boldsymbol{H}$ [4] or by solution of the normal equations of this problem which are

$$
\boldsymbol{H}^{T} \boldsymbol{H b}=-\boldsymbol{H}^{T} \hat{h}
$$

In order to determine $c$, one proceeds as follows. We can rewrite (2) as

$$
\left[c^{T} O^{T}\right]^{T}=B g
$$

where

$$
\begin{aligned}
\boldsymbol{c} & =\left[c_{0} c_{1} \cdots c_{N}\right]^{T} \\
\boldsymbol{g} & =\left[\begin{array}{llll}
g_{0} g_{1} & \cdots & g_{n_{0}-1}
\end{array}\right]^{T} \\
\boldsymbol{B} & =\left[\begin{array}{llll}
1 & 0 & \cdots & 0 \\
b_{1} & 1 & \cdots & 0 \\
\vdots & & & \vdots \\
b_{M} & b_{M-1} & \cdots & 0 \\
0 & b_{M} & \cdots & 0 \\
\vdots & & & \vdots \\
0 & 0 & \cdots & 1
\end{array}\right]
\end{aligned}
$$

$\boldsymbol{B}$ is thus an $n_{0} \times n_{0}$ lower triangular Toeplitz nonsingular matrix. One now defines a second error measure $e_{k}$ where

$$
e_{k}=g_{k}-h_{k} \quad 0 \leqslant k \leqslant n_{0}-1,
$$

or in vector notation

$$
e=g-h
$$

where

$$
\begin{aligned}
\boldsymbol{e} & =\left[e_{0} e_{1} \cdots e_{n_{0}-1}\right]^{T} ; \\
\boldsymbol{h} & =\left[h_{0} h_{1} \cdots h_{n_{0}-1}\right]^{T} .
\end{aligned}
$$

Substituting for $g$ in (6), we get

$$
\left[c^{T} O^{T}\right]^{T}=B(h+e) \text {. }
$$

Since $\boldsymbol{B}$ is nonsingular, it has an inverse $\boldsymbol{B}^{-1}$, and so

$$
\boldsymbol{h}+\boldsymbol{e}=\boldsymbol{B}^{-1}\left[\boldsymbol{c}^{T} O^{T}\right]^{T} .
$$

Denoting the first $N+1$ columns of $\boldsymbol{B}^{-1}$ as the matrix $\boldsymbol{W}$, we have

$$
\boldsymbol{h}+\boldsymbol{e}=W_{c}
$$


Although not explicitly mentioned in [2], the elements of the matrix $W$ can easily be obtained from the first $n_{0}$ points of the impulse response $u(n)$ of $1 / B(z)$. To see this, we note that

$$
B u=\left[\begin{array}{ll}
1 & O^{T}
\end{array}\right]^{T}
$$

where

$$
\boldsymbol{u}=\left[u(0) u(1) \cdots u\left(n_{0}-1\right)\right]^{T} .
$$

So,

$$
\boldsymbol{u}=\boldsymbol{B}^{-1}\left[1 O^{T}\right]^{T} .
$$

Now since $\boldsymbol{B}$ is lower triangular Toeplitz, $\boldsymbol{B}^{-1}$ is also lower triangular Toeplitz, and is therefore completely characterized by its first column. Therefore, $\boldsymbol{W}$ is also completely characterized by its first column. So if

$$
\boldsymbol{W}=\left[w_{i j}\right],
$$

then

$$
w_{i j}=w_{i-j+1,1}=u(i-j) \quad 1 \leqslant i \leqslant n_{0}, 1 \leqslant j \leqslant N+1 .
$$

(Note: $u(n)=0$ if $n<0$.)

Burrus and Parks now suggest five different methods for solving $c$ from (8). These are briefly outlined below.

1) The first $N+1$ outputs are made exact, i.e., $g(k)=h(k)$ or $e(k)=0$ for $k=0,1, \cdots, N$. In this case, the first $N+1$ rows of (8) can be used to solve for $c$.

2) Any $N+1$ outputs are made exact. Here the corresponding $N+1$ rows of $\boldsymbol{W}$ are used to form a square matrix which, if nonsingular, is inverted to give $c$.

3) The norm of $\boldsymbol{e}$ is minimized. Here $\boldsymbol{c}$ is obtained by solving the normal equations which are

$$
\boldsymbol{w}^{T} \boldsymbol{W c}=\boldsymbol{W}^{T} \boldsymbol{h} \text {. }
$$

This is similar to Shanks' method [8].

4) The weighted norm of $\boldsymbol{e}$ is minimized. Thus, if the weighted norm is $e^{T} T e$ where $T$ is a positive definite matrix, then $c$ is obtained by solving the normal equations which are

$$
\boldsymbol{W}^{T} \boldsymbol{T} \boldsymbol{W} \boldsymbol{c}=\boldsymbol{W}^{T} \boldsymbol{T h} .
$$

5) A combination of methods 1 and 3 can be formulated by requiring that a certain number of terms $L$, less than $N+1$, in $e(k)$ be zero, and the $N+1-L$ remaining degrees of freedom be used to minimize the norm of $e$. This is a quadratic minimization problem with equality constraints which results in linear equations for $\boldsymbol{c}$.

This concludes our review of the Burrus-Parks algorithm.

\section{Modeling of Aliased Signal}

In this section, we describe the model used for a general noncausal signal. We then show how the aliased data can be related to this model. Finally, we discuss the simplifications that result when the signal is purely causal or purely anticausal.

A noncausal signal $s(n)$ can be represented as the sum of a causal and an anticausal part. We denote the causal part as $s_{+}(n)$ and the time-reversed anticausal part as $s_{-}(n)$. Thus,

$$
\begin{aligned}
s_{+}(n) & =s(n) & & n \geqslant 0 \\
& =0 & & n<0 ; \\
s_{-}(n) & =s(-n) & & n>0 \\
& =0 & & n \leqslant 0 .
\end{aligned}
$$

We now model $s_{+}(n)$ as the impulse response of a stable, causal filter $S_{+}(z)$, where

$$
S_{+}(z)=\frac{\alpha(z)}{P(z)}=\frac{\sum_{i=0}^{N_{1}} \alpha_{i} z^{-i}}{1+\sum_{i=1}^{M_{1}} p_{i} z^{-i}} .
$$

We further assume that the rational model is a proper rational model, and so $N_{1}<M_{1}$. Since the sequence $s_{-}(n)$ starts from $n=1$, we model it as the impulse response of a filter $S_{-}(z)=$ $z^{-1} S_{-1}(z)$ where $S_{-1}(z)$ is assumed to be a stable, causal filter and is expressed as

$$
S_{-1}(z)=\frac{\beta(z)}{Q(z)}=\frac{\sum_{i=0}^{N_{2}} \beta_{i} z^{-i}}{1+\sum_{i=1}^{M_{2}} q_{i} z^{-i}} .
$$

Again we assume that the rational model is a proper rational model, and so $N_{2}<M_{2}$. The impulse response $s_{-1}(n)$ of the filter $S_{-1}(z)$ is related to $s_{-}(n)$ by

$$
s_{-}(n)=s_{-1}(n-1) \text {. }
$$

The signal $s(n)$ can itself be expressed as the impulse response of $S(z)$, where

$$
\begin{aligned}
S(z) & =S_{+}(z)+S_{-}\left(z^{-1}\right) \\
& =S_{+}(z)+z S_{-1}\left(z^{-1}\right) .
\end{aligned}
$$

The aliased data $h(n)$ can now be expressed as

$$
\begin{aligned}
h(n) & =\sum_{k=-\infty}^{+\infty} s\left(n+k n_{0}\right) \\
& =\sum_{k=0}^{\infty} s_{+}\left(n+k n_{0}\right)+\sum_{k=0}^{\infty} s_{-}\left(n_{0}-n+k n_{0}\right) \\
& =\sum_{k=0}^{\infty} s_{+}\left(n+k n_{0}\right)+\sum_{k=0}^{\infty} s_{-1}\left(n_{0}-n-1+k n_{0}\right) \\
& \quad \text { for all } n=0,1, \cdots, n_{0}-1 .
\end{aligned}
$$

In order to see how the aliased data can be related to the signal model, we define the causal sequences

$$
\begin{array}{ll}
h_{1}(n)=\sum_{k=0}^{\infty} s_{+}\left(n+k n_{0}\right) & \text { for all } n \geqslant 0 \\
h_{2}(n)=\sum_{k=0}^{\infty} s_{-1}\left(n+k n_{0}\right) & \text { for all } n \geqslant 0 .
\end{array}
$$


If we assume that $S_{+}(z)$ has only simple poles $\left\{z_{i}\right\}$ with corresponding residues $\left\{A_{i}\right\}[7]$, then

$$
s_{+}(n)=\sum_{i=1}^{M_{1}} A_{i} z_{i}^{n} \quad \text { for all } n \geqslant 0 .
$$

So,

$$
\begin{aligned}
h_{1}(n) & =\sum_{k=0}^{\infty} s_{+}\left(n+k n_{0}\right) \\
& =\sum_{i=0}^{M_{1}} A_{i} \sum_{k=0}^{\infty} z_{i}^{n+k n_{0}} \\
& =\sum_{i=1}^{M_{1}} \frac{A_{i} z_{i}^{n}}{1-z_{i}^{n_{0}}} \\
& =\sum_{i=1}^{M_{1}} \bar{A}_{i} z_{i}^{n} \quad \text { for all } n \geqslant 0
\end{aligned}
$$

where

$$
\bar{A}_{i}=\frac{A_{i}}{1-z_{i}{ }^{n_{0}}} .
$$

Thus, $h_{1}(n)$ can be modeled as the impulse response of a filter $H_{1}(z)$ which has the same poles $\left\{z_{i}\right\}$ but different residues $\left\{\bar{A}_{i}\right\}$. We conclude that

$$
H_{1}(z)=\sum_{i=1}^{M_{1}} \frac{\bar{A}_{i}}{1-z_{i} z^{-1}}=\frac{\bar{\alpha}(z)}{P(z)}
$$

where $\bar{\alpha}(z)$ is the new numerator polynomial whose degree is $\leqslant M_{1}-1$ and $P(z)$ is the original denominator of $S_{+}(z)$. The same conclusions can be reached by a simple argument even when $S_{+}(z)$ has multiple poles. For example, if $S_{+}(z)$ has a pole at $z=z_{i}$ of multiplicity $r$, then the contribution of this pole $s_{+}^{(i)}(n)$ to the impulse response $s_{+}(n)$ can be expressed as

$$
s_{+}^{(i)}(n)=\sum_{j=1}^{r} A_{i_{j}} n^{r-j_{i}} z_{i}^{n}
$$

So the contribution to $h_{1}(n)$ is given by

$$
\begin{aligned}
h_{1}^{(i)}(n) & =\sum_{k=0}^{\infty} s_{+}^{(i)}\left(n+k n_{0}\right) \\
& =\sum_{k=0}^{\infty} \sum_{j=1}^{r} A_{i_{j}}\left(n+k n_{0}\right)^{r-j} z_{i}^{n+k n_{0}} .
\end{aligned}
$$

This can be expressed in the same form as $s_{+}^{(i)}(n)$, i.e.,

$$
h_{1}^{(i)}(n)=\sum_{j=1}^{r} \bar{A}_{i_{j}} n^{r-j} z_{i}^{n} .
$$

This implies that $H_{1}(z)$ also has a pole at $z=z_{i}$ of multiplicity $r$. So, in general, $H_{1}(z)$ has poles of the same order and at the same locations as the poles of $S_{+}(z)$. So both $H_{1}(z)$ and $S_{+}(z)$ have the same denominator polynomial. The numerator polynomial is different since the residues corresponding to the dif- ferent poles have changed. Similarly, we can model $h_{2}(n)$ as the impulse response of a filter $H_{2}(z)$ which has the same denominator $Q(z)$ as $S_{-1}(z)$, but a different numerator polynomial $\bar{\beta}(z)$ which is of degree $\leqslant M_{2}-1$. Thus,

$$
H_{2}(z)=\frac{\bar{\beta}(z)}{Q(z)} \text {. }
$$

We can now express the aliased data $h(n)$ in terms of $h_{1}(n)$ and $h_{2}(n)$ using (15)-(17).

$$
\begin{aligned}
& h(n)=h_{1}(n)+h_{2}\left(n_{0}-n-1\right) \\
& \quad \text { for all } n=0,1, \cdots, n_{0}-1 .
\end{aligned}
$$

Thus, the aliased data $h(n)$ can be modeled as the first $n_{0}$ points (i.e., from $n=0$ to $n=n_{0}-1$ ) of the impulse response of the filter $H(z)$ where

$$
\begin{aligned}
H(z) & =H_{1}(z)+z^{-\left(n_{0}-1\right)} H_{2}\left(z^{-1}\right) \\
& =\frac{\bar{\alpha}(z)}{P(z)}+z^{-\left(n_{0}-1\right)} \frac{\bar{\beta}\left(z^{-1}\right)}{Q\left(z^{-1}\right)} \\
& =\frac{\bar{C}(z)}{B(z)}
\end{aligned}
$$

where

$$
\begin{aligned}
& \bar{C}(z)=z^{-M_{2}}\left[\bar{\alpha}(z) Q\left(z^{-1}\right)+z^{-\left(n_{0}-1\right)} \bar{\beta}\left(z^{-1}\right) P(z)\right] / q_{M_{2}} \\
& B(z)=1+\sum_{i=1}^{M} b_{i} z^{-i}=z^{-M_{2}} P(z) Q\left(z^{-1}\right) / q_{M_{2}} .
\end{aligned}
$$

Clearly, $M=M_{1}+M_{2}$. (Note: As long as $q_{M_{2}} \neq 0$, which will be the case if $M_{2}$ is not overestimated, the normalization by $q_{M_{2}}$ will not affect the final result in the ideal noise-free case.)

We have thus shown that if the signal $s(n)$ is modeled as in (12)-(14), then the aliased data $h(n)$ can be viewed as the first $n_{0}$ points of the impulse response of a filter $H(z)$ defined in (26)-(28). The denominator or predictor polynomial of $H(z)$ is simply a delayed and scaled version of the product of the predictor polynomials of the rational models $S_{+}(z)$ and $S_{-1}\left(z^{-1}\right)$ which represent the causal and anticausal parts of the signal $s(n)$.

We now consider two special cases: 1) A purely causal signal, and 2) a purely anticausal signal. In the first case, $s_{-}(n)=0$ for all $n$, and so $h(n)$ is simply equal to $h_{1}(n)$ which is defined in (16). Thus, the aliased data $h(n)$ are simply the first $n_{0}$ points of the impulse response of the filter $H_{1}(z)$. We therefore conclude that

$$
\begin{aligned}
H(z) & =H_{1}(z) \\
\bar{C}(z) & =\bar{\alpha}(z) \\
B(z) & =P(z) .
\end{aligned}
$$

These equations can also be obtained from (26)-(28) by substituting $\beta(z)=0, \bar{\beta}(z)=0, M_{2}=0, Q(z)=1$, and $q_{M_{2}}=q_{0}=$ 1. In the second case of a purely anticausal signal, $s_{+}(n)=0$ for all $n$, and so $h(n)$ is simply equal to $h_{2}(n)$ which is defined 
in (17). Thus, the aliased data are simply the first $n_{0}$ points of the impulse response of $z^{-\left(n_{0}-1\right)} H_{2}\left(z^{-1}\right)$. We therefore conclude that

$$
\begin{aligned}
& H(z)=z^{-\left(n_{0}-1\right)} H_{2}\left(z^{-1}\right) \\
& \bar{C}(z)=z^{-M_{2}} z^{-\left(n_{0}-1\right)} \bar{\beta}\left(z^{-1}\right) / q_{M_{2}} \\
& B(z)=z^{-M_{2}} Q\left(z^{-1}\right) / q_{M_{2}} .
\end{aligned}
$$

Again, these can be obtained from (26)-(28) by substituting $\alpha(z)=0, \bar{\alpha}(z)=0, M_{1}=0$, and $P(z)=1$.

\section{Estimation of the Predictor Polynomials}

In this section, we describe an algorithm for estimating the predictor polynomial $B(z)$ of the filter $H(z)$. We will also show how to determine the degree of the predictor polynomial. Methods to obtain $P(z)$ and $Q\left(z^{-1}\right)$, the predictor polynomials which characterize the causal and anticausal parts of the signal $s(n)$, are then described.

We recall that the filter $H(z)$ is given by

$$
H(z)=\frac{\bar{C}(z)}{B(z)}
$$

where

$$
\begin{aligned}
& \bar{C}(z)=z^{-M_{2}}\left[\bar{\alpha}(z) Q\left(z^{-1}\right)+z^{-\left(n_{0}-1\right)} \bar{\beta}\left(z^{-1}\right) P(z)\right] / q_{M_{2}} \\
& B(z)=1+\sum_{i=1}^{M} b_{i} z^{-i}=z^{-M_{2}} P(z) Q\left(z^{-1}\right) / q_{M_{2}} .
\end{aligned}
$$

On examining the numerator polynomial $\bar{C}(z)$, we find that it has nonzero coefficients of $z^{-i}$ from $j=0$ to $j=M-1$ and from $j=n_{0}$ to $j=n_{0}+M-1$. Thus, if the modeling were exact, then the coefficients of $z^{-j}$ from $j=M$ to $j=n_{0}-1$ will be zero. Expressing this as a convolution, we have

$$
h(i)+\sum_{j=1}^{M} b_{j} h(i-j)=\epsilon_{i} \quad i=M, M+1, \cdots, n_{0}-1
$$

where the error terms $\epsilon_{i}$ have been introduced to take into account the fact that the modeling may not be exact. Expressing (29) in matrix form, we have

$$
\hat{\boldsymbol{h}}+\boldsymbol{H b}=\boldsymbol{\epsilon}
$$

where

$$
\begin{aligned}
& \boldsymbol{H}=\left[\begin{array}{llll}
h(M-1) & h(M-2) & \cdots & h(0) \\
h(M) & h(M-1) & \cdots & h(1) \\
h\left(n_{0}-2\right) & h\left(n_{0}-3\right) & \cdots & h\left(n_{0}-M-1\right)
\end{array}\right] ; \\
& \hat{\boldsymbol{h}}=\left[h(M) h(M+1) \cdots h\left(n_{0}-1\right)\right]^{T} ; \\
& \boldsymbol{b}=\left[b_{1} b_{2} \cdots b_{M}\right]^{T} ; \\
& \boldsymbol{\epsilon}=\left[\epsilon_{M} \epsilon_{M+1} \cdots \epsilon_{n_{0}-1}\right]^{T} .
\end{aligned}
$$

Thus, $\boldsymbol{H}$ is an $\left(n_{0}-M\right) \times M$ matrix, $\hat{\boldsymbol{h}}$ and $\epsilon$ are $\left(n_{0}-M\right) \times 1$ vectors, and $\boldsymbol{b}$ is an $M \times 1$ vector. One can immediately see the similarity between (30) and (4) in the Burrus-Parks algorithm. If $n_{0} \geqslant 2 M$, the vector $b$ which minimizes $\epsilon^{T} \epsilon$ can be uniquely obtained by solving the resulting normal equations, which are

$$
H^{T} H b=-H^{T} \hat{h}
$$

or

$$
R \boldsymbol{b}=-\boldsymbol{x}
$$

where the $(i, j)$ element of $r_{i j}$ of the $M \times M$ symmetric nonnegative definite matrix $\boldsymbol{R}=\boldsymbol{H}^{T} \boldsymbol{H}$ is given by

$$
r_{i j}=\sum_{k=0}^{n_{0}-M-1} h(M-i+k) h(M-j+k)
$$

and the $i$ th element $x_{i}$ of the vector $\boldsymbol{x}=\boldsymbol{H}^{T} \hat{\boldsymbol{h}}$ is given by

$$
x_{i}=\sum_{k=0}^{n_{0}-M-1} h(M-i+k) h(M+k) .
$$

The normal equations in (31) are identical to the normal equations obtained in LPC [14]. Efficient algorithms for solving LPC normal equations involving only $O\left(M^{2}\right)$ arithmetic operations have been described by Morf et al. [18]. The Cholesky decomposition [4] is another well-known technique for solving these normal equations.

Another approach for obtaining $\boldsymbol{b}$ which minimizes $\epsilon^{T} \epsilon$ is to use the SVD (i.e., singular value decomposition) of $\boldsymbol{H}$. SVD techniques have been used for ARMA modeling in spectral estimation [17]. This approach is known to have superior numerical properties [4]. In addition, it can be used to obtain the degree of the predictor polynomial $B(z)$. We will now describe how this can be done.

Let us assume that the predictor polynomial is exactly of degree $M$. Then from (29), we have

$$
h(i)+\sum_{j=1}^{M} b_{j} h(i-j)=0 \quad i=M, M+1, \cdots, n_{0}-1 .
$$

(The error terms $\epsilon_{i}=0$ for all $M \leqslant i \leqslant n_{0}-1$ since the modeling is exact.) If we overestimate the degree of the predictor polynomial as $M+1$, then the matrix $\boldsymbol{H}^{(M+1)}$, which is the matrix $\boldsymbol{H}$ when the degree of $B(z)$ is $M+1$, takes the form

$$
\boldsymbol{H}^{(M+1)}=\left[\begin{array}{llll}
h(M) & h(M-1) & \cdots & h(0) \\
h(M+1) & h(M) & \cdots & h(1) \\
h\left(n_{0}-2\right) & h\left(n_{0}-3\right) & \cdots & h\left(n_{0}-M-2\right)
\end{array}\right] .
$$

But from (32), we see that the columns of the matrix $\boldsymbol{H}^{(M+1)}$ are all dependent, and $\boldsymbol{H}^{(M+1)}$ is therefore rank deficient. Conversely, if $\boldsymbol{H}^{(M+1)}$ is rank deficient and is of rank $M$, then there exists constants $t_{0}, t_{1}, \cdots, t_{M}$ such that

$$
\sum_{i=0}^{M} t_{i} h(j-i)=0 \quad j=M, M+1, \cdots, n_{0}-2 .
$$


All constants $t_{i}$ must be nonzero, for otherwise $\boldsymbol{H}^{(M+1)}$ would be of rank lower than $M$, which contradicts the assumption. So dividing by $t_{0}$, we get

$$
h(j)+\sum_{i=1}^{M} \frac{t_{i}}{t_{0}} h(j-i)=0 \quad j=M, M+1, \cdots, n_{0}-2
$$

But this set of equations is exactly satisfied if the degree of the predictor polynomial were $M$. We therefore conclude that the matrix $\boldsymbol{H}$ is rank deficient if the degree of the predictor polynomial is overestimated. A good indicator of rank deficiency is the lowest singular value of the matrix. So one can determine the degree of the predictor polynomial as follows. We monitor the lowest singular value of $\boldsymbol{H}$ as we increase the value of $M$. When it becomes less than or equal to a certain threshold for some $M^{*}$, the procedure is terminated and the degree of the predictor polynomial is chosen to be $M^{*}-1$.

Having determined $B(z)$, we now focus our attention on determining $P(z)$, the predictor polynomial which characterizes the causal part of the signal, and $Q\left(z^{-1}\right)$, the predictor polynomial which characterizes the anticausal part of the signal. The polynomials $P(z), Q\left(z^{-1}\right), B(z)$ are related by the equation

$$
B(z)=z^{-M_{2}} P(z) Q\left(z^{-1}\right) / q_{M_{2}} .
$$

Since the rational models $S_{+}(z)$ and $S_{-}(z)$ representing the causal and the anticausal parts of the signal are assumed to be stable, the roots of $P(z)$ lie within the unit circle and the roots of $Q\left(z^{-1}\right)$ lie outside the unit circle. Thus, $P(z)$ becomes the minimum-phase part of $B(z)$ and $Q\left(z^{-1}\right)$ becomes its maximum-phase part $[6, c h .7]$. Thus, the problem reduces to finding the minimum-phase and maximum-phase parts of $B(z)$.

One approach is to find all the roots of $B(z)$. We then group all the roots inside the unit circle to form the minimum-phase part and all the roots outside the unit circle (except the roots at infinity) to form the maximum-phase part. But this would involve many computations, especially when $M$ is very large. An alternative is to use the complex cepstrum $\hat{b}(n)$ of $B(z)$ [i.e., the inverse Fourier transform of $\left.\log B\left(e^{j \omega}\right)\right]$. The complex cepstrum $\hat{b}(n)$ would be, in general, two-sided or noncausal since $B(z)$ is a mixed-phase polynomial (see $[6$, sect. 10.5.1]). We can think of $\hat{b}(n)$ as being made up of four parts.

1) Complex cepstrum $\hat{p}(n)$ of $P(z)$ which is zero for all $n<0$ since $P(z)$ is minimum phase [6, sect. 10.5.1] .

2) Complex cepstrum $\hat{q}(n)$ of $Q\left(z^{-1}\right)$ which is zero for all $n>0$ since $Q\left(z^{-1}\right)$ is maximum phase [6, sect. 10.5.1].

3) Complex cepstrum of the constant $1 / q_{M_{2}}$ which only affects $\hat{b}(0)$.

4) Complex cepstrum of the delay term $z^{-M_{2}}$ which is equal to $-M_{2} \cos (\pi n) / n$ for $n \neq 0$ and zero if $n=0$ [19].

The effect of delay $z^{-M_{2}}$ can easily be removed and the value of $M_{2}$ can be easily estimated as follows. The unwrapped phase of $B\left(e^{j \omega}\right)$, which we need to compute before we can compute the complex cepstrum, consists of two parts. One contribution is from the delay $z^{-M_{2}}$ and it is equal to $-j \omega M_{2}$. The other part is due to $P(z)$ and $Q\left(z^{-1}\right)$ and it is periodic with period $2 \pi$. By estimating the slope of the linear ramp in the unwrapped phase, we can determine $M_{2}$. To remove the contribution of $z^{-M_{2}}$ to the complex cepstrum of $B(z)$, we simply subtract this linear ramp from the unwrapped phase. So in practice, one really computes $\hat{b}_{M_{2}}(n)$, the complex cepstrum of $z^{M_{2}} B(z)$, and it is equal to $\hat{p}(n)$ for all $n>0$ and $\hat{q}(n)$ for all $n<0$.

The minimum-phase polynomial $P(z)$ and the maximumphase polynomial $Q(z)$ can now be estimated by using the following recursion formulas (see [6, sect. 10.5.2] for a derivation):

$$
\begin{array}{rlrl}
p_{n} & =1 & n=0 \\
& =\hat{b}_{M_{2}}(n)+\sum_{k=1}^{n-1} \frac{k}{n} p_{n-k} \hat{b}_{M_{2}}(k) & 1 \leqslant n \leqslant M_{1} \\
q_{n} & =1 & & n=0 \\
& =\hat{b}_{M_{2}}(-n)+\sum_{k=1}^{n-1} \frac{k}{n} q_{n-k} \hat{b}_{M_{2}}(-k) & & 1 \leqslant n \leqslant M_{2} .
\end{array}
$$

We conclude this section with the following observation. If we have some a priori knowledge about the model orders (i.e., $M, M_{1}$, or $M_{2}$ ), then the algorithm can be considerably simplified. For example, if we knew that the signal was causal, then all that we need compute is $B(z)$ for we know that, in this case, $P(z)=B(z)$. Again, if we knew that the signal was purely anticausal, then we only need compute $B(z)$ for $B(z)=$ $z^{-M_{2}} Q\left(z^{-1}\right) / q_{M_{2}}, M_{2}=M$, and so $Q(z)=z^{-M} B\left(z^{-1}\right) / b_{M}$.

\section{Estimation of the Numerator Polynomials}

In this section, we will describe the methods to obtain the numerator polynomials $\alpha(z)$ and $\beta(z)$ of the rational models $S_{+}(z)$ and $S_{-1}(z)$. We will also consider the special cases when the signal is purely causal or purely anticausal. Our approach will be similar to that of Burrus and Parks.

We first find the impulse response $\{u(n)\}$ of the filter $1 / P(z)$ and the impulse response $v(n)$ of the filter $1 / Q(z)$. Then

$$
\begin{aligned}
s_{+}(n) & =\sum_{j=0}^{\min \left(N_{1}, n\right)} \alpha_{j} u(n-j) \\
s_{-1}(n) & =\sum_{j=0}^{\min \left(N_{2}, n\right)} \beta_{j} v(n-j) .
\end{aligned}
$$

So substituting in (15) which was

$$
\begin{gathered}
h(n)=\sum_{k=0}^{\infty} s_{+}\left(n+k n_{0}\right)+\sum_{k=0}^{\infty} s_{-1}\left(n_{0}-n-1+k n_{0}\right) \\
0 \leqslant n \leqslant n_{0}-1,
\end{gathered}
$$

we get

$$
\begin{aligned}
h(n)= & \sum_{j=0}^{N_{1}} \alpha_{j} \sum_{k=0}^{\infty} u\left(n+k n_{0}-j\right)+\sum_{j=0}^{N_{2}} \beta_{j} \sum_{k=0}^{\infty} \\
& \cdot v\left(n_{0}-n-1+k n_{0}-j\right) \quad 0 \leqslant n \leqslant n_{0}-1 .
\end{aligned}
$$


[Note: Both $u(n)$ and $v(n)$ are taken to be zero for all $n<0$ in (41).] In the presence of modeling errors, (41) must be modified to incorporate the error terms. Thus, (41) becomes

$$
\begin{aligned}
h(n)+e(n)= & \sum_{j=0}^{N_{1}} \alpha_{j} \sum_{k=0}^{\infty} u\left(n+k n_{0}-j\right) \\
& +\sum_{j=0}^{N_{2}} \beta_{j} \sum_{k=0}^{\infty} v\left(n_{0}-n-1+k n_{0}-j\right) \\
& \quad 0 \leqslant n \leqslant n_{0}-1 .
\end{aligned}
$$

In matrix form, this becomes

$$
w c=h+e
$$

where $\boldsymbol{W}$ is an $n_{0} \times\left(N_{1}+N_{2}+2\right)$ matrix with elements

$$
\begin{aligned}
& w_{i j}= \sum_{k=0}^{\infty} u\left(i-j+k n_{0}\right) \\
& 1 \leqslant i \leqslant n_{0}, \quad 1 \leqslant j \leqslant N_{1}+1 \\
&=\sum_{k=0}^{\infty} v\left(n_{0}-i+k n_{0}-\left(j-N_{1}-2\right)\right) \\
& \quad 1 \leqslant i \leqslant n_{0}, \quad N_{1}+2 \leqslant j \leqslant N_{1}+N_{2}+2
\end{aligned}
$$

and the vectors $\boldsymbol{c}, \boldsymbol{h}$, and $\boldsymbol{e}$ are defined as

$$
\begin{aligned}
\boldsymbol{c} & =\left[\alpha_{0} \alpha_{1} \cdots \alpha_{N_{1}} \beta_{0} \beta_{1} \cdots \beta_{N_{2}}\right]^{T} ; \\
\boldsymbol{h} & =\left[h(0) h(1) \cdots h\left(n_{0}-1\right)\right]^{T} ; \\
\boldsymbol{e} & =\left[e(0) e(1) \cdots e\left(n_{0}-1\right)\right]^{T} .
\end{aligned}
$$

One immediately observes the similarity between (43) and (8) in the Burrus-Parks method. But the matrices $W$ are different in both cases. In the Burrus-Parks formulation, the elements of $\boldsymbol{W}$ are given by (9), but in the present case they are given by (43a). It is this difference that takes into account the fact that we are dealing with the aliased version of the signal rather than the signal itself. One can now solve for $c$ by any of the five indirect methods suggested by Burrus and Parks. The degrees of $\alpha(z)$ and $\beta(z)$ (i.e., $N_{1}$ and $N_{2}$ ) are taken to be the lowest values in the ranges $\left[0, M_{1}-1\right]$ and $\left[0, M_{2}-1\right]$, respectively, for which we get satisfactory results.

In the special case of a purely causal signal, we only need determine $\alpha(z)$ since $\beta(z)=0$. So here the matrix $W$ is an $n_{0} X$ $\left(N_{1}+1\right)$ matrix with elements

$$
w_{i j}=\sum_{k=0}^{\infty} u\left(i-j+k n_{0}\right) \quad 1 \leqslant j \leqslant n_{0}, 1 \leqslant j \leqslant N_{1}+1 .
$$

and the vector $c$ is defined as

$$
c=\left[\alpha_{0} \cdots \alpha_{N_{1}}\right]^{T} \text {. }
$$

If the signal were a purely anticausal signal, we only need determine $\beta(z)$ since $\alpha(z)=0$. So here the matrix $\boldsymbol{W}$ is an $n_{0} \times$ $\left(N_{2}+1\right)$ matrix with elements

$$
\begin{aligned}
w_{i j}=\sum_{k=0}^{\infty} v\left(n_{0}-i+k n_{0}-j+1\right) \\
1 \leqslant i \leqslant n_{0}, 1 \leqslant j \leqslant N_{2}+1
\end{aligned}
$$

and the vector $c$ is defined as

$$
c=\left[\beta_{0} \cdots \beta_{N_{2}}\right]^{T} \text {. }
$$

This concludes our description of the algorithm for obtaining the dealiased signal. A brief outline of the various steps in the algorithm is given below.

Step 1: Find the predictor polynomial $B(z)$ by minimizing $\epsilon^{T} \epsilon$ in (30).

Step 2: Factorize $B(z)$ to obtain $P(z)$ and $Q\left(z^{-1}\right)$. This factorization can be done using the complex cepstrum of $B(z)$.

Step 3: Find the impulse response $u(n)$ of the filter $1 / P(z)$ and the impulse response $v(n)$ of the filter $1 / Q(z)$.

Step 4: Set up the numerator-determining set of equations $\boldsymbol{W} \boldsymbol{c}=\boldsymbol{h}+\boldsymbol{e}$ where $\boldsymbol{W}, \boldsymbol{c}, \boldsymbol{h}$, and $\boldsymbol{e}$ are as defined in (43a), (43b), $(43 \mathrm{c})$, and (43d), respectively. Solve for the vector $c$ by any of the five indirect methods of Burrus and Parks. This determines the numerator polynomials $\alpha(z)$ and $\beta(z)$.

Step 5: Find $s_{+}(n)$, the impulse response of the filter $S_{+}(z)=$ $\alpha(z) / P(z)$. Find $s_{-}(n)$, the impulse response of the filter $S_{-}(z)=$ $z^{-1} \beta(z) / Q(z)$. Then the dealiased signal $s(n)$ is given by

$$
\begin{array}{rlrl}
s(n) & =s_{+}(n) & & n \geqslant 0 \\
& =s_{-}(-n) & & n<0 . \\
& & \text { V. Simulation Results }
\end{array}
$$

\section{Simulation Results}

In order to test the algorithm, the following two experiments were performed. In both of the experiments, the numerator polynomials were determined using the third indirect method of Burrus and Parks, i.e., by minimizing $\boldsymbol{e}^{T} \boldsymbol{e}$ in (43).

In the first experiment, the signal $s(n)$ was taken to be the impulse response of a stable, causal filter $S(z)$. The value of $n_{0}$ in our simulation was taken to be 50 . The aliased data $h(n)$ were then generated from $n=0$ to $n=n_{0}-1$ using $h(n)=\Sigma_{k=0}^{\infty}$ $s\left(n+k n_{0}\right)$. The waveforms of the estimated signal $\hat{s}(n)$ and the estimated aliased waveform $\hat{h}(n)$ are overlaid by the original signal $s(n)$ and the original aliased waveform $h(n)$ and are displayed in Fig. 1(a) and (b), respectively. The error waveforms $\hat{s}(n)-s(n)$ and $\hat{h}(n)-h(n)$ are magnified by a factor of 100 and are displayed in Fig. 1(c) and (d).

In the second experiment, the signal $s(n)$ was generated using two causal sequences $s_{+}(n)$ and $s_{-}(n)$. The causal sequences were taken to be the impulse response of filters $S_{+}(z)$ and $S_{-}(z)$, respectively. The signal $s(n)$ is then given by

$$
\begin{aligned}
s(n) & =s_{+}(n) & & n \geqslant 0 \\
& =s_{-}(-n) & & n<0 .
\end{aligned}
$$

The value of $n_{0}$ was again chosen to be 50 in our simulation. The aliased data were then generated from $n=0$ to $n=n_{0}-1$ using $h(n)=\Sigma_{k=-\infty}^{\infty} s\left(n+k n_{0}\right)$. The waveforms of the estimated signal $\hat{s}(n)$ and the estimated aliased waveform $\hat{h}(n)$ are overlaid by the original $s(n)$ and the original aliased waveform $h(n)$ and are displayed in Fig. 2(a) and (b), respectively. The error waveforms $\hat{s}(n)-s(n)$ and $\hat{h}(n)-h(n)$ are magnified by a factor of 100 and are displayed in Fig. 2(c) and (d). It can be seen from the simulation results in both of the experiments that the signal can be recovered with excellent accuracy. 


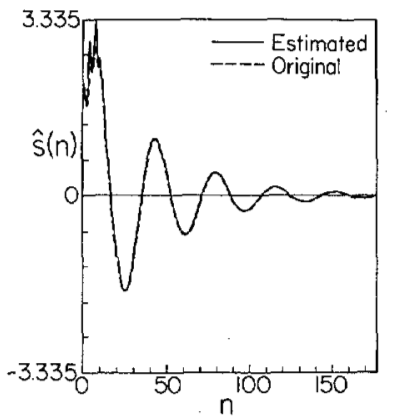

(a) Estimated signal (overlaid by original)

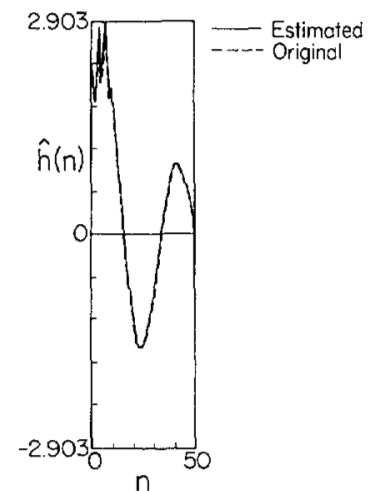

(b) Estimated aliased data

(overloid by original aliosed data)

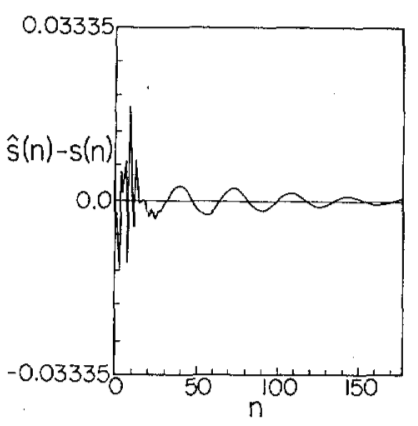

(c) Error in the estimated signo

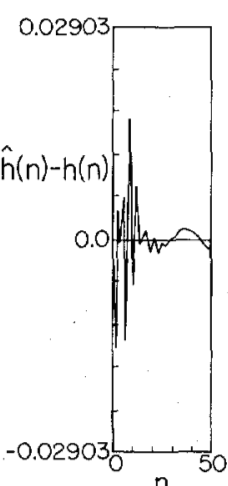

(d) Error in the estimated aliased data

Fig. 1. Simulation results for the case of causal signals.

\section{Summary and CONClusions}

In this paper, the problem of signal restoration from its aliased version was considered. Models for the signal were developed and algorithms for estimating the parameters of these

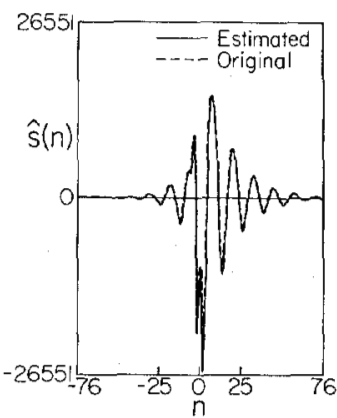

(a) Estimated signal (overlaid by original)

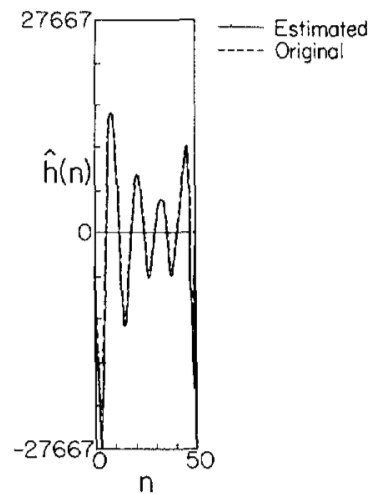

(b) Estimated aliosed data (overlaid by original aliased data)

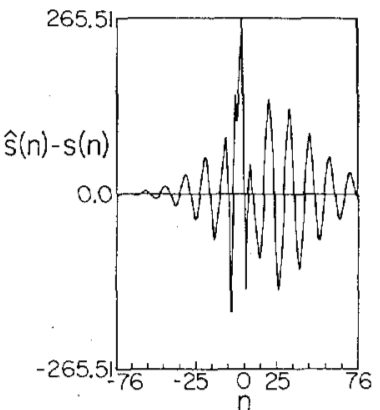

(c) Error in the estimated signal

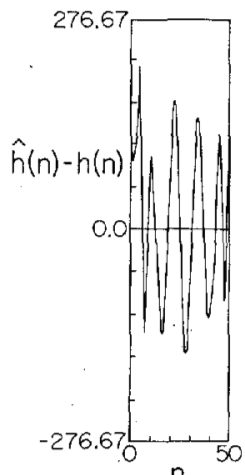

(d) Error in the estimated aliased dato

Fig. 2. Simulation results for noncausal signals.

modeis from the aliased data were described. These algorithms only involved solving linear equations. Simulation tests were conducted, and it was shown that the signal can be recovered very accurately. We have thus demonstrated that by modeling 
the signal suitably and estimating the parameters of the model, one can recover the signal from its aliased version with very little loss in accuracy. In doing so, we have extended the earlier works of Burrus and Parks.

A closely related problem is the problem of system identification from the system response to multipulse input, which may not necessarily be periodic. The motivation for such a problem comes from recent papers in speech modeling [23] which have shown that speech can be effectively modeled as the output of a frequency-shaping filter when driven by a multipulse input. It is not very difficult to see that the filter parameters can be estimated using similar techniques described in this paper if we knew what the amplitudes and locations of the input pulses were. But the problem becomes much more involved when the locations of the pulses are themselves unknown. We are currently investigating methods for estimating the filter parameters as well as the excitation parameters simultaneously.

\section{ACKNOWLEDGMENT}

The author wishes to express his sincere thanks to Prof. E. C. Posner for helpful suggestions during the course of this work and for reviewing this paper. The author would also like to express his deep appreciation and gratitude to the three anonymous reviewers whose suggestions have considerably improved earlier versions of this paper.

\section{REFERENCES}

[1] R. J. Marks, II, "Restoration of continuously sampled band limited signals from aliased data," IEEE Trans. Acoust., Speech, Signal Processing, vol. ASSP-30, pp. 937-941, Dec. 1982.

[2] C. S. Burkus and T. W. Parks, "Time domain design of digital filters," IEEE Trans. Audio Electroacoust., vol. AU-18, pp. $138-142,1970$

[3] C. E. Fröberg, Introduction to Numerical Analysis. Reading, MA: Addison-Wesley, 1965.

[4] C. L. Lawson and R. J. Hanson, Solving Least Squares Problems. Englewood Cliffs, NJ: Prentice-Hall, 1974.

[5] A. Papoulis, Signal Analysis. New York: McGraw-Hill, 1977.

[6] A. V. Oppenheim and R. W. Schafer, Digital Signal Processing. Englewood Cliffs, NJ: Prentice-Hall, 1975.

[7] R. V. Churchill, Complex Variables and Applications, 2nd ed. New York: McGraw-Hill, 1960.

[8] J. L. Shanks, "Recursion filters for digital processing," Geophys, vol. 32, pp. 33-51, Feb. 1967

[9] R. N. McDonough and W. H. Huggins, "Best least squares representation of signals by exponentials," IEEE Trans. Automat. Contr., vol. AC-13, pp. 408-412, Aug. 1968.

[10] F. Brophy and A. C. Salazar, "Recursive digital fiiter synthesis in the time domain," IEEE Trans. Acoust., Speech, Signal Processing, vol. ASSP-22, pp. 45-55, Feb. 1974.

[11] - "Consideration of the Padé approximant technique in the synthesis of recursive digital filters," IEEE Trans. Audio Electroacoust., vol. AU-21,pp. 500-505, Dec. 1973.

[12] C. T. Mullis and R. A. Roberts, "The use of second order information in the approximation of discrete time linear systems," IEEE Trans. Acoust., Speech, Signal Processing, vol. ASSP-24, pp. 226-238, June 1976.

[13] R. Hastings-James and S. K. Mehra, "Extensions of the Padé approximant technique for the design of recursive digital filters," IEEE Trans. Acoust., Speech, Signal Processing, vol, ASSP-25, pp. 501-509, Dec. 1977.

[14] J. Makhoul, "Linear prediction: A tutorial review," Proc. IEEE, vol. 63 , pp. 561-580, Apr. 1975.

[15] J. D. Markel and A. H. Gray, Jr, Linear Prediction of Speech. Berlin, Germany: Springer, 1976.

[16] E. A. Robinson, "Predictive decomposition of time series with applications to seismic exploration," Geophys., vol. 32, pp. 418484,1967

[17] J. A. Cadzow, "Spectral estimation: An overdetermined rational model equation approach," Proc. IEEE, vol. 70, pp. 907-939, Sept. 1982.

[18] M. Morf, B. Dickinson, T. Kailath, and A. Viera, "Efficient solutions of covariance equations for linear prediction," IEEE Trans. Acoust., Speech, Signal Processing, vol. ASSP-25, pp. 429-433, Oct. 1979.

[19] A. V. Oppenheim and R. W. Schafer, "Homomorphic analysis of speech," IEEE Trans. Audio Electroacoust., vol. AU-16, pp. 221-226, June 1968

[20] A. V. Oppenheim, R. W. Schafer, and T. G. Stockham, Jr., "Nonlinear filtering of multiplied and convolved signals," Proc. IEEE, vol. 56, pp. 1264-1291, Aug. 1968 .

[21] J. L. Flanagan, Speech Analysis, Synthesis and Perception, 2nd ed. New York: Springer-Verlag, 1972.

[22] L. R. Rabiner and R. W. Schafer, Digital Processing of Speech Signals. Englewood Cliffs, NJ: Prentice-Hall, 1978.

[23] B. S. Atal and J. R. Remde, "A new model of LPC excitation for producing natural-sounding speech at low bit rates," in Proc. ICASSP '82, pp. 614-617.

[24] J. M. Tribolet, "A new phase unwrapping algorithm," IEEE Trans. Acoust., Speech, Signal Processing, vol. ASSP-25, pp. 170177, Apr. 1977.

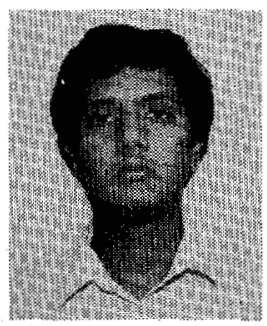

Kumar Swaminathan (S'81-S'83) was born in India on April 11, 1959. He received the B.Tech. degree in 1981 from the Indian Institute of Technology, Madras, and the M.S. degree in 1982 from the California Institute of Technology, Pasadena, where he is presently working towards the Ph.D. degree, all in electrical engineering.

His areas of interest are digital signal processing; speech processing, and linear systems.

Mr. Swaminathan is a member of Sigma Xi 